\title{
Effectiveness of L-thyroxine treatment on TSH suppression during pregnancy in patients with a history of thyroid carcinoma after total thyroidectomy and radioiodine ablation
}

\author{
Blaz Krhin ${ }^{1}$ and Nikola Besic ${ }^{2}$ \\ ${ }^{1}$ Department of Laboratory Diagnostics, Institute of Oncology Ljubljana, Ljubljana, Slovenia \\ ${ }^{2}$ Department of Surgical Oncology, Institute of Oncology Ljubljana, Ljubljana, Slovenia
}

Radiol Oncol 2012; 46(2): 160-165.

Received 29 September 2011

Accepted 24 November 2011

Correspondence to: Prof. Nikola Bešič MD, PhD, Dept. of Surgical Oncology, Institute of Oncology Ljubljana, Zaloška 2, SI-1000 Ljubljana, Slovenia. Phone: +386 15879 953; Fax:+386 1 5879400; E-mail: nbesic@onko-i.si

Disclosure: No potential conflicts of interest were disclosed.

Introduction. There are scarce data about the optimal increase of L-thyroxine dose during pregnancy in patients with a history of thyroid carcinoma. The first aim of the study was to find out if routine therapeutic measures enable adequate TSH suppression in pregnancy. The other aim was to find out the optimal dose of L-thyroxine for TSH suppression in pregnant women.

Patients and methods. In this retrospective observational study, we analysed 36 pregnancies of 32 women with a history of thyroid carcinoma. Before pregnancy, all of them underwent total thyroidectomy and radioiodine ablation of thyroid remnant, and they were on suppressive doses of L-thyroxine. Thyroid function tests were obtained before, during and after pregnancy.

Results. Mean L-thyroxine dose before pregnancy, in the first, second and, third trimester and after delivery was 149 , 147, 155, 165 and 158 micrograms daily, respectively. TSH concentration remained suppressed in 9 pregnancies, it was within normal range in 22 and elevated in 5 pregnancies. The mean dose of L-thyroxine in patients with suppressed TSH before pregnancy, in the first, second and, third trimester and after delivery was 154, 154, 164, 160 and 161 micrograms daily, respectively. When the dose had to be changed, the mean increase of the dose was 31.5 micrograms daily. Conclusions. The range of changes in TSH concentration during pregnancy in the patients who have been on suppressive L-thyroxine therapy before conception is quite wide. TSH was adequately suppressed in only $25 \%$ of pregnancies. The dose of L-thyroxine in patients with suppressed TSH in the first, second and third trimester was 154, 164 and 160 micrograms daily, respectively.

Key words: pregnancy; TSH suppression; L-thyroxine; thyroid carcinoma.

\section{Introduction}

Thyroid hormones are important for normal pregnancy and the foetal development. ${ }^{1,2}$ During pregnancy, maternal thyroid hormones requirements increase. ${ }^{3,4}$ It is well known that the reference values of TSH, free $\mathrm{T}_{3}$ and free $\mathrm{T}_{4}$ for healthy the nonpregnant population are not the same as during pregnancy. Dashe et al. ${ }^{5}$ published the data about the TSH concentration in 13,599 pregnancies. They found out that the normal physiological concen- tration of TSH during the first trimester of pregnancy was as low as $0.1 \mathrm{mU} / \mathrm{L}$. According to the trimester-specific reference ranges of serum, TSH concentrations above $2.3 \mathrm{mU} / \mathrm{L}$ in the first trimester and 3.1-3.5 $\mathrm{mU} / \mathrm{L}$ in the second and third trimester may already be indicative of subclinical hypothyroidism. ${ }^{1,6}$ Safety of pregnancy during subclinical hyperthyroidism was reported by Casey et al. 7 They found out that the subclinical hyperthyroidism was not associated with the adverse outcome of pregnancy. ${ }^{7}$ 
The patients having undergone thyroidectomy and radioiodine therapy are dependent on exogenous L-thyroxine. ${ }^{8}$ Some authors advocate that the increase of dose of L-thyroxine during pregnancy should be determined from the results of thyroid function tests ${ }^{8-11}$, while others propose to increase L-thyroxine dose as soon as pregnancy is confirmed. ${ }^{12,13}$

There are very limited data in the literature about the changes of TSH and thyroid hormones during pregnancy in patients with a history of thyroid carcinoma. To our knowledge, these studies included only a small number of patients ${ }^{8,12-15}$ with the largest study group of 18 such cases reported by Loh et al. ${ }^{8}$ There are scarce data about the optimal increase of L-thyroxine dose during pregnancy in the patients with thyroid carcinoma after total thyroidectomy and radioiodine ablation of thyroid remnant. The first aim of the study was to find out if routine therapeutic measures enable adequate TSH suppression in pregnancy. The other aim was to find out the optimal dose of L-thyroxine for TSH suppression in pregnant women.

\section{Material and methods}

In this retrospective observational study we analysed 36 pregnancies of 32 women (mean age at conception $29.9 \pm 0.6$ years) with a history of thyroid carcinoma during the period from 2000 to 2009. T1, $\mathrm{T} 2$ and $\mathrm{T} 3$ tumour was diagnosed in 15,7 and $10 \mathrm{pa}-$ tients, respectively. In 23 and 9 patients, the tumour stage was assessed as $\mathrm{N} 0$ and N1, respectively. None of the patients had distant metastases. All of them had no evidence of disease at conception and after delivery. Histopathology of bioptic specimen revealed the presence of Hashimoto's thyroiditis in $11 / 36(=31 \%)$ of patients. Before pregnancy, all of them underwent total thyroidectomy and radioiodine ablation of the thyroid remnant and they were all on suppressive doses of L-thyroxine. All patients were advised to take L-thyroxine four hours before vitamins, iron or calcium drugs.

At conception, the age of patients was 22 to 37 years (mean age $29.9 \pm 0.6$ years). Among our patients, 28 women were pregnant once and four twice. Pregnancy passed without any events in 30 cases and with complications in six cases (premature delivery in two cases, preeclampsia in one, increased blood pressure in one, high serum glucose concentration in two, vaginal bleeding in the seventh month of gestation in one case).
Thyroid function tests were performed before, during and after pregnancy. The last test of thyroid function before pregnancy was done 1-12 (median 3) months before conception. During pregnan$\mathrm{cy}$, a clinical exam and thyroid function tests were performed every 6-8 weeks. When more than one set of tests was performed in any one trimester, the highest TSH concentration with the corresponding free $T_{3}$ and free $T_{4}$ concentrations were used in the statistical analysis in order to minimize any bias towards avoidance of dose change. The median number of thyroid function tests performed during pregnancy in each woman was four (range3-6). L-thyroxine dose was modified to maintain serum TSH below $0.3 \mathrm{mU} / \mathrm{L}$. Suppression dose was adequate if TSH was $0.01-0.29 \mathrm{mU} / \mathrm{L}$ and free $\mathrm{T}_{3}$ was within normal range.

From 2000-2006, TSH was measured by the two-site immunoluminometric assay (sandwich principle) LIASON TSH (Byk-Sangtec Diagnostica, Dietzenbach, Germany). Free $\mathrm{T}_{3}$ and free $\mathrm{T}_{4}$ were measured by commercially available kits (LIAISON $\mathrm{FT}_{3}$, LIAISON $\mathrm{FT}_{4}$ ) with "LIAISON" Immunoassay System (Byk-Sangtec, Germany later DiaSorin, Italy). Reference values for TSH, free $\mathrm{T}_{3}$ and free $\mathrm{T}_{4}$ were $0.27-4.2 \mathrm{mU} / \mathrm{L}, 2.93-6.8 \mathrm{pmol} / \mathrm{L}$ and 7.7-23.2 pmol/L, respectively. From 2007 onwards, $T S H$, free $\mathrm{T}_{3}$ and free $\mathrm{T}_{4}$ were measured by commercially available kits (TSH, $\mathrm{FT}_{3}, \mathrm{FT}_{4}$ ) with "Modular Analytics E170" Immunoassay System (Roche Diagnostics, Mannheim, Germany). Reference values for TSH, free $\mathrm{T}_{3}$ and free $\mathrm{T}_{4}$ were 0.27-4.20 mIU/L, 3.1-6.8 pmol/L and 12-22 pmol/L, respectively.

The study was reviewed by the appropriate medical ethics committee. The Institute's Protocol Review Board approved the study, which was performed in accordance with the medical ethics standards laid down in an appropriate version of the 1964 Declaration of Helsinki. The participants gave informed consent.

\section{Statistical analysis}

Changes in the results of thyroid-function tests, and L-thyroxine doses throughout pregnancy were analysed by repeated measures ANOVA or Friedman's test, followed by Wilcoxon signed rank test in case of non-normal data distribution. P-values of less than 0.05 were considered to indicate statistical significance. The software package SPSS 16.0 for Windows (SPSS Inc., Chicago, IL USA) was used. 


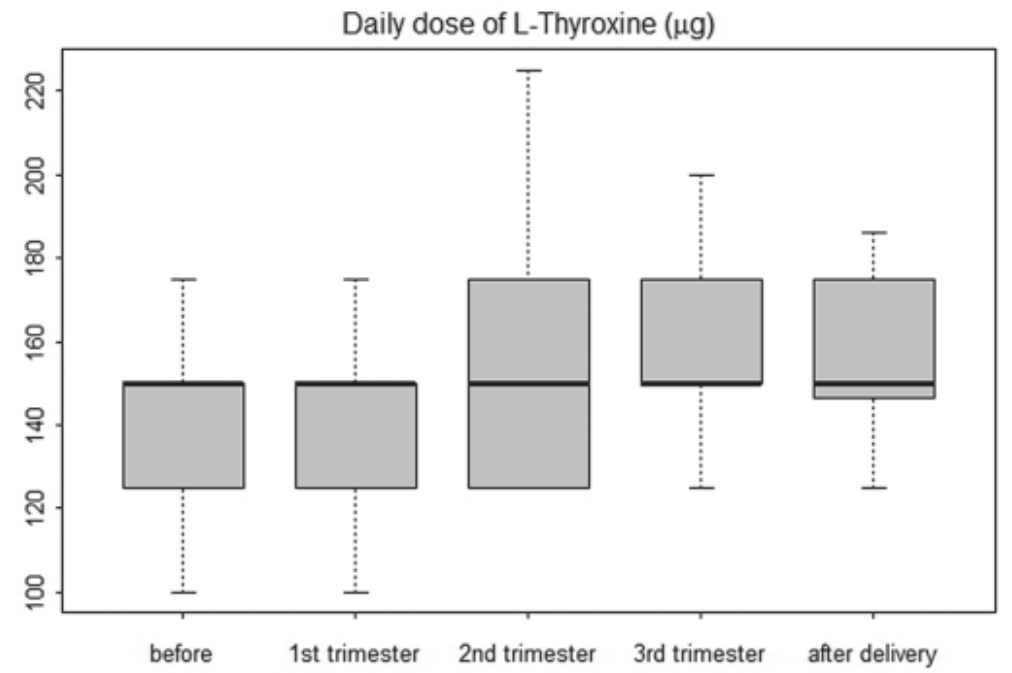

FIGURE 1. Changes of TSH during 36 pregnancies. The bottom and top of the box are the 25th and 75th percentile (the lower and upper quartiles, respectively), and the band near the middle of the box is the 50th percentile (the median). The ends of the whiskers represent the minimum and maximum of all the data.

\section{$\mathrm{TSH}(\mathrm{mU} / \mathrm{l})$}

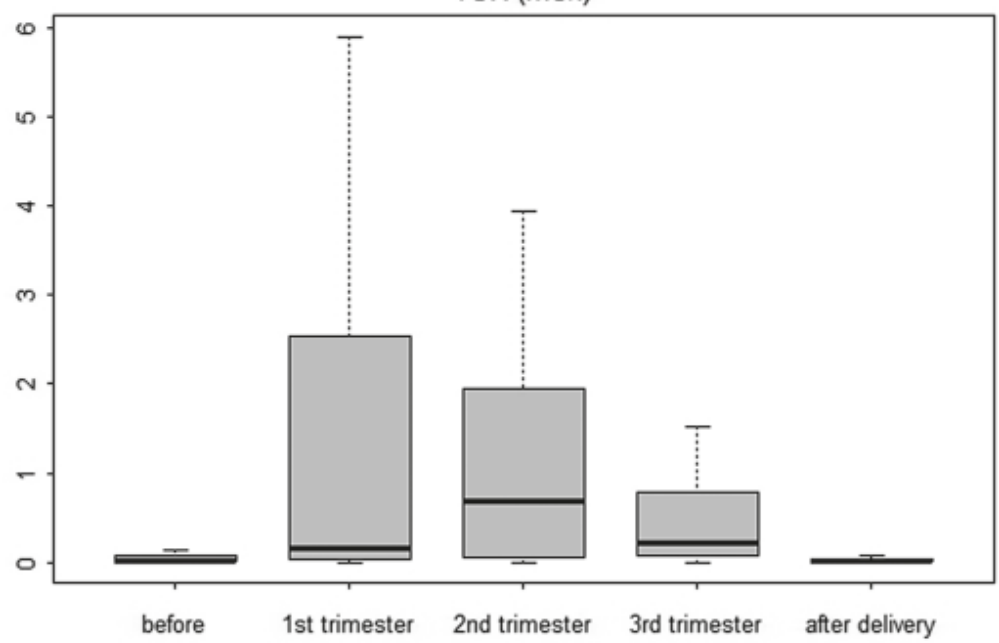

FIGURE 2. Changes of dose of L-thyroxine during 36 pregnancies. The bottom and top of the box are the 25th and 75th percentile (the lower and upper quartiles, respectively), and the band near the middle of the box is the 50th percentile (the median). The ends of the whiskers represent the minimum and maximum of all the data.

\section{Results}

The mean concentration of $\mathrm{TSH}$, free $\mathrm{T}_{4}$ and free $\mathrm{T}_{3}$ before pregnancy, in the first, second and third trimester and after delivery are presented in Table 1. The concentration of TSH during the first, second and third trimester were higher in comparison to the TSH concentration before pregnancy $(\mathrm{p}<0.001)$. The changes of TSH concentration during pregnancy are presented in Figure 1. In none of our patients, a decrease of TSH was observed in the first 16 weeks of pregnancy.

The doses of L-thyroxine during the second and the third trimester were higher than the doses before pregnancy $(p<0.05)$. The changes of dose of L-thyroxine during pregnancy are presented in Figure 2. Obese patients had larger dose of L-thyroxine in comparison to patients with normal body mass index (BMI) or underweight patients before conception as well as during pregnancy. However, BMI had no impact on the proportion of patients with adequate TSH suppression during pregnancy. The time period from the last test before pregnancy to the first test in pregnancy did not differ in women with adequate TSH suppression and women with inadequate TSH suppression $(\mathrm{p}=0.377)$.

Changes of the mean TSH concentration and dose of L-thyroxine before and during pregnancy and after delivery in patients with suppressed and not suppressed TSH concentration are presented in Table 2. The mean dose of L-thyroxine in patients with suppressed TSH before pregnancy, in the first, second and, third trimester and after delivery was 154, 154, 164, 160 and 161 micrograms daily, respectively.

In 36 pregnancies, the TSH concentration remained suppressed during 9 pregnancies. In 22 pregnancies the TSH concentration was within normal range, whilein5 pregnancies it was elevated. A dose of L-thyroxine was not changed in 14 pregnancies (mean dose 159 micrograms daily); TSH was suppressed in 5 pregnancies and within normal range in 9 pregnancies.

The dose of L-thyroxine had to be increased in 22 pregnancies. The TSH concentration in 22 pregnancies remained suppressed in 4, within normal range in 13 and elevated in 5 of them. TSH was over $5.0 \mathrm{mU} / \mathrm{L}$ during the first trimester and second trimester in 5/22 (23\%) cases and 3/22 (14\%) cases, respectively.

The dose of L-thyroxine was increased 30 times and decreased four times during the course of pregnancy. The dose was changed 9, 11 and 14 times in the second, third and the fifth month, respectively. The mean increase of L-thyroxine dose in these 22 cases was 32.15 (range 25-75) micrograms.

\section{Discussion}

According to the current American Thyroid Association and European Thyroid Association guidelines $^{16,17}$, the patients with a history of thyroid 
TABLE 1. Mean L-thyroxine dose and mean TSH concentration before pregnancy, in the first trimester, the second trimester, the third trimester and after delivery.

\begin{tabular}{|c|c|c|c|c|}
\hline & $\begin{array}{l}\text { Daily dose of L-thyroxine } \\
\text { Mean ( } \pm \mathrm{SD} \text {; range) } \\
\text { Micrograms }\end{array}$ & $\begin{array}{l}\text { Mean TSH ( } \pm S D ; \text { range) } \\
\mathrm{mU} / \mathrm{L}\end{array}$ & FT4 ( \pm SD; range) pmol/L & FT3 ( \pm SD; range) pmol/L \\
\hline Before pregnancy & $149( \pm 32 ; 100-300)$ & $0.07( \pm 0.12 ; 0.001-0.29)$ & $20.13( \pm 5.08 ; 13.9-28.4)$ & $5.16( \pm 0.86 ; 3.91-6.61)$ \\
\hline 1. trimester & $147( \pm 34 ; 100-300)$ & $1.96( \pm 3.60 ; 0.001-14.54)$ & $16.72( \pm 4.32 ; 9.29-27.6)$ & $4.15( \pm 1.08 ; 2.41-7.6)$ \\
\hline 2. trimester & $155( \pm 35 ; 125-300)$ & $1.43( \pm 1.96 ; 0.001-7.64)$ & $14.79( \pm 3.39 ; 8.95-24.1)$ & $4.00( \pm 0.85 ; 2.72-5.5)$ \\
\hline 3. trimester & $165( \pm 34 ; 125-300)$ & $0.63( \pm 0.95 ; 0.001-4.60)$ & $14.04( \pm 3.15 ; 5.07-23.1)$ & $3.83( \pm 0.69 ; 2.52-5.2)$ \\
\hline
\end{tabular}

carcinoma are either on substitution or suppressive doses of L-thyroxine. Our study group consisted of 36 pregnancies in 32 patients with a history of thyroid carcinoma. Before conception they were treated by total thyroidectomy and radioiodine ablation of thyroid remnant and were on the suppressive dose of L-thyroxine. So, in all of our patients there was no viable thyroid tissue to produce thyroid hormones. These patients were, therefore, entirely dependent on exogenous L-thyroxine.

In healthy women, a transient decrement of serum TSH concentration occurs during the first two months of pregnancy, which is a result of the elevation of human chorionic gonadotropin (hCG) having similar molecular structure as TSH. ${ }^{1,18,19}$ In none of our patients, lower concentration of TSH was detected during the first two months of pregnancy. In the absence of viable thyroid tissue, there was no effect of elevated concentration of hCG on the synthesis and secretion of thyroid hormones from thyroid and on the consequent decrease of TSH concentration.

According to Endocrine Society Clinical Practice Guideline ${ }^{1}$, L-thyroxine dose often needs to be increased within 4-6 weeks of gestation and may require a $30-50 \%$ addition of dosage. ${ }^{3,4}$ But we observed that there was no need to increase the dose during pregnancy in $25 \%$ of our cases who were on suppressive doses of L-thyroxine before conception. Also Loh et al. ${ }^{8}$ observed that there was no need to change the dosage in some of the patients with thyroid carcinoma who were on TSH suppression therapy before pregnancy. Based on our results, we believe that an increment of dose immediately after conception as advocated by some authors ${ }^{12}$ is not appropriate for all the patients who are on suppressive doses of L-thyroxine. We agree with Loh at al. ${ }^{8}$ that these patients may be over treated by an empiric increase of L-thyroxine dose and may develop overt hyperthyroidism.
The other important finding of our study is that TSH suppression was not achieved in $75 \%$ of cases when thyroid testing was performed each 6-8 weeks. Furthermore, an elevation of TSH level above normal was observed in $14 \%$ of our patients. Possibly, this could be avoided, if thyroid function tests would be obtained every 4-6 weeks as recommended by Endocrine Society Clinical Practice Guideline $^{1}$, or every 4 weeks as recommended by Yassa et $a l . .^{13}$ In a recent study Yassa et al. ${ }^{13}$ performed serum testing every two weeks until the $20^{\text {th }}$ week of pregnancy and at the $30^{\text {th }}$ week of pregnancy. If blood samples were obtained every four weeks, $92 \%$ of abnormal TSH concentrations were detected. But, if an every six week testing protocol had been followed, only $73 \%$ of abnormal TSH concentrations would have been detected..$^{10}$ So, they concluded that the thyroid function tests should be repeated every four weeks during the first half of pregnancy.

It is still not known when it is most appropriate to increase the dose of L-thyroxine ${ }^{20}$ : before pregnancy ${ }^{21}$, immediately after conception ${ }^{12,13}$ or when elevation of TSH is observed. ${ }^{8-11}$ Rotondi et al..$^{21}$ suggested the increase of L-thyroxine dose to "partially suppressive" dose before pregnancy. The patients who underwent thyroidectomy because of multinodular goitre or Hashimoto's thyroiditis and who were on substitution therapy were randomized in two groups before conception: one group continued with substitution doses, while the other was on partially suppressive dose. ${ }^{21}$ Before conception, the average dose in the substitution group and the partially suppressive group of patients was 143 and 178 micrograms, while the average TSH was 1.84 and $0.48(0.32-0.7) \mathrm{mU} / \mathrm{L}$, respectively. ${ }^{21} \mathrm{TSH}$ level above $3.5 \mathrm{mU} / \mathrm{L}$ was found to be more common in the substitution group in comparison to the "partially suppressive" group of patients (36\% vs. $14 \%)$. 
TABLE 2. Adequacy of suppression, mean L-thyroxine dose and mean TSH concentration before pregnancy, in the first trimester, the second trimester, the third trimester and after delivery.

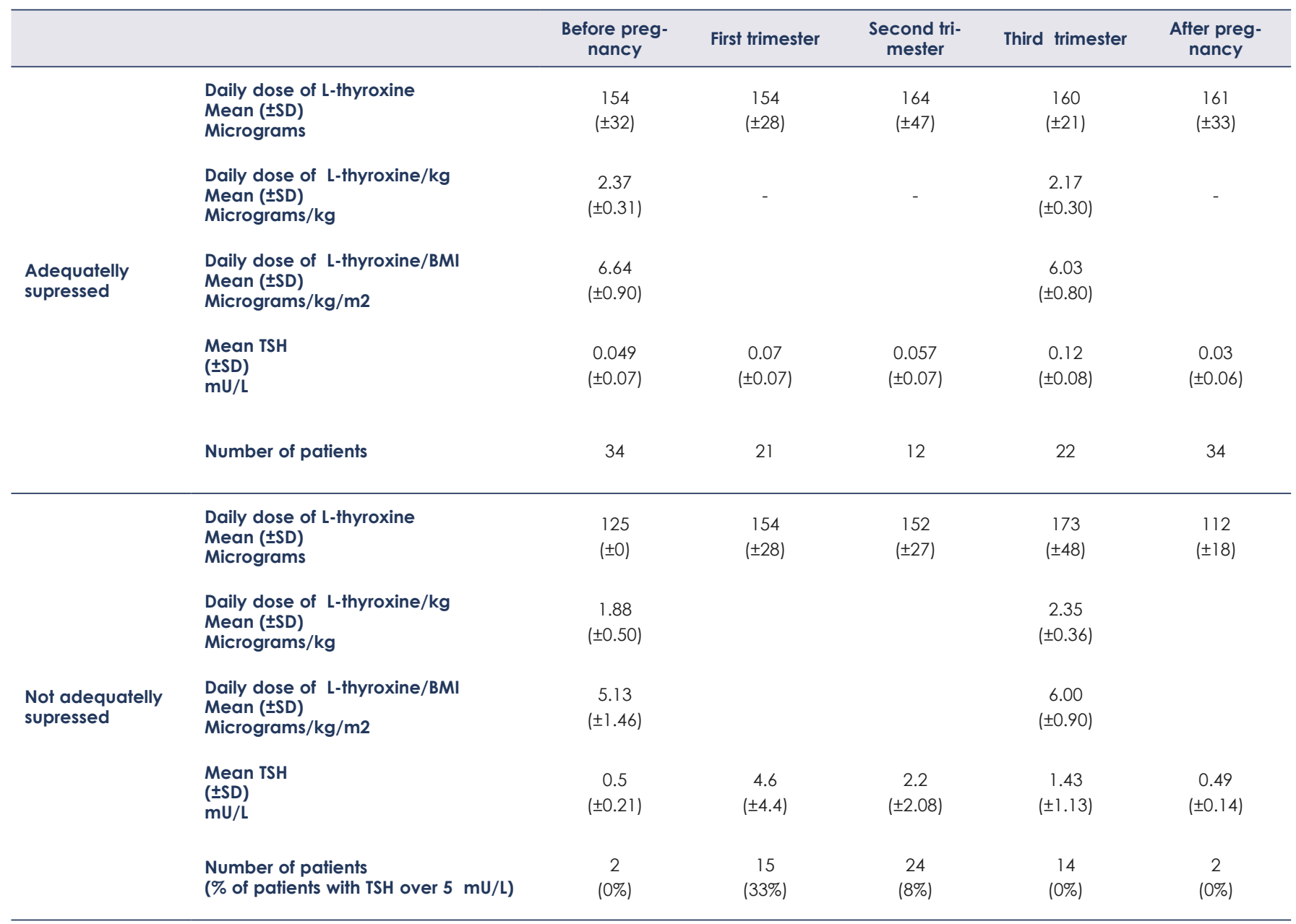

Another approach, i.e. increment of the dose as soon as pregnancy is confirmed, was proposed by Alexander et al.. ${ }^{12}$ They studied precisely the timing and amount of L-thyroxine adjustment in 20 pregnancies in 19 women. ${ }^{12}$ Eight patients had Hashimoto's disease, six thyroid carcinoma, three Graves disease and two were after the treatment for benign thyroid nodule. L-thyroxine requirements increased as early as the fifth week of gestation. ${ }^{12}$ That is why they recommended an increase of L-thyroxine dose as soon as possible. ${ }^{12}$ Their opinion is that the dose should be increased by about $30 \% .{ }^{12}$ Yassa et al. ${ }^{13}$ performed a prospectively randomized study in which women were receiving an increased L-thyroxine dose at the beginning of pregnancy. The dose in the first group was increased by $29 \%$, and in the second group, by $43 \%$. The mean dose before and after the change in the first and the second group was 112, 145, 109 and
156 micrograms, respectively. ${ }^{13}$ After the change of dose, TSH suppression was present in $32 \%$ of women from the first group and in $65 \%$ of women from the second group; but less than $0.1 \mathrm{mU} / \mathrm{L}$ of TSH was found in only one patient $(8 \%)$ from the first group and in six patients from the second group $(26 \%) .{ }^{13}$ They found out that a $29 \%$ increase of dose (from 112 to 145 micrograms) prevented maternal TSH elevation over 2.5 and $5.0 \mathrm{mU} / \mathrm{L}$ in $85 \%$ and $100 \%$ of patients, respectively. But the majority of their patients had Hashimoto's disease, while our patients had a history of thyroid carcinoma and were without any functional thyroid tissue. In our patients, the mean dose before pregnancy was 149 micrograms of L-thyroxine. When a dosage during pregnancy remained the same, the mean dose was 159 micrograms. This dosage prevented the elevation of TSH over $5.0 \mathrm{mU} / \mathrm{L}$ in all the cases. On the other hand, when a dosage during pregnancy had 
to be changed, the mean dose before conception was 125 micrograms only. In the latter cases, TSH was over $5.0 \mathrm{mU} / \mathrm{L}$ during the first and second trimester in $23 \%$ of cases and $14 \%$, respectively.

The third approach, increment of a dose based on thyroid function tests was reported by Loh et al. ${ }^{8}$ In 18 cases with a history of thyroid carcinoma, the average daily L-thyroxine dose before conception was 153 micrograms. During pregnancy they required an increase of dose by $26 \% .^{8}$ In our patients, the average dose was 149 micrograms before conception. But during pregnancy our patients required an increase of dose by $11 \%$ only. Obviously the range of changes in TSH concentration during pregnancy in the patients who have been on suppressive L-thyroxine therapy before conception is quite wide.

As a conclusion, the patients with thyroid carcinoma who are on high doses of L-thyroxine require close monitoring of thyroid function tests during pregnancy. TSH was adequately suppressed in only $25 \%$ of pregnancies. The dose of L-thyroxine in patients with suppressed TSH in the first, second and third trimester was 154, 164 and $160 \mathrm{mi}-$ crograms daily, respectively. When the dose had to be changed, the mean increase of the dose was 31.5 (range 25-75) micrograms daily.

\section{Acknowledgments}

Research studies P3-0289 supported by the Ministry of Education, Science and Sport of Slovenia. This work was presented in part at the $14^{\text {th }}$ International Thy roid Congress, held in Paris, France, September 11-16 2011.
7. Casey BM, Dashe JS, Wells CE, McIntire DD, Leveno KJ, Cunningham FG. Subclinical hyperthyroidism and pregnancy outcomes. Obstet Gynecol 2006; 107: 337-41.

8. Loh JA, Wartofsky L, Jonklaas J, Burman KD. The magnitude of increased levothyroxine requirements in hypothyroid pregnant women depends upon the etiology of the hypothyroidism. Thyroid 2009; 19: 269-75.

9. Girling JC, de Swiet M. Thyroxine dosage during pregnancy in women with primary hypothyroidism. Br J Obstet Gynaecol 1992; 99: 368-70.

10. Verga U, Bergamaschi S, Cortelazzi D, Ronzoni S, Marconi AM, Beck-Peccoz P. Adjustment of L-T4 substitutive therapy in pregnant women with subclinical, overt or post-ablative hypothyroidism. Clin Endocrinol (Oxf) 2009; 70: 798-802

11. Hallengren B, Lantz M, Andreasson B, Grennert L. Pregnant women on thyroxine substitution are often dysregulated in early pregnancy. Thyroid 2009; 19: 391-4.

12. Alexander EK, Marqusee E, Lawrence J, Jarolim P, Fischer GA, Larsen PR. Timing and magnitude of increases in levothyroxine requirements during pregnancy in women with hypothyroidism. N Engl J Med 2004; 351: 241-9.

13. Yassa L, Marqusee E, Fawcett R, Alexander EK. Thyroid hormone early adjustment in pregnancy (the THERAPY) trial. J Clin Endocrinol Metab 2010; 95: 3234-41.

14. Chopra IJ, Baber K. Treatment of primary hypothyroidism during pregnancy: is there an increase in thyroxine dose requirement in pregnancy? Metabolism 2003; 52: 122-8.

15. Dobrenic M, Huic D, Zuvic M; Grosev D, Petrovic R, Samardzic T. Usefulness of low iodine diet in managing patients with differentiated thyroid cancer initial results. Radiol Oncol 2011; 45: 189-95.

16. Cooper DS, Doherty GM, Haugen BR, Kloos RT, Lee SL, Mandel SJ, et al. Management guidelines for patients with thyroid nodules and differentiated thyroid cancer. Thyroid 2006; 16: 109-142.

17. Pacini F, Schlumberger $M$, Dralle $H$, Elisei $R$, Smit JW, Wiersinga W. The European Thyroid Cancer Taskforce: European consensus for the management of patients with differentiated thyroid carcinoma of the follicular epithelium. Europ J Endocrinol 2006; 154: 787-803.

18. Glinoer D. The regulation of thyroid function in pregnancy: pathways of endocrine adaptation from physiology to pathology. Endocr Rev1997; 18: 404-33.

19. Gaberscek S, Zaletel K. Thyroid physiology and autoimmunity in pregnancy and after delivery. Expert Rev Clin Immunol 2011;7: 697-707.

20. Toft A. Increased levothyroxine requirements in pregnancy--why, when, and how much? N Engl J Med 2004; 351: 292-4.

21. Rotondi M, Mazziotti G, Sorvillo F, Piscopo M, Cioffi M, Amato G, et al. Effects of increased thyroxine dosage pre-conception on thyroid function during early pregnancy. Eur J Endocrinol 2004; 151: 695-700.

\section{References}

1. Abalovich M, Amino N, Barbour LA, Cobin RH, De Groot L, Glinoer D, et al. Management of thyroid dysfunction during pregnancy and postpartum: an Endocrine Society Clinical Practice Guideline. J Clin Endocrinol Metab 2007; 92: S1-47.

2. Haddow JE, Palomaki GE, Allan WC, Williams JR, Knight GJ, Gagnon J, et al. Maternal thyroid deficiency during pregnancy and subsequent neuropsychological development of the child. N Engl J Med 1999; 341: 549-55.

3. Kaplan MM. Monitoring thyroxine treatment during pregnancy. Thyroid 1992; 2: 147-52

4. Mandel SJ, Larsen PR, Seely EW, Brent GA. Increased need for thyroxine during pregnancy in women with primary hypothyroidism. $N$ Engl J Med 1990; 323: 91-6.

5. Dashe JS, Casey BM, Wells CE, McIntire DD, Byrd EW, Leveno KJ, et al. Thyroid-stimulating hormone in singleton and twin pregnancy: importance of gestational age-specific reference ranges. Obstet Gynecol 2005; 106: 753-7.

6. Panesar NS, Li CY, Rogers MS. Reference intervals for thyroid hormones in pregnant Chinese women. Ann Clin Biochem 2001; 38: 329-32. 